\title{
Current Solutions for Long-Segment Tracheal Reconstruction
}

\author{
Ahmed A. Abouarab, MD, MRCS, ${ }^{1}$ Hany H. Elsayed, MD, FRCS, Cth, ${ }^{2}$ Hussein Elkhayat, MD, ${ }^{3}$
} Ahmed Mostafa, MD, ${ }^{4}$ David C. Cleveland, MD, ${ }^{5}$ and Ahmed El Nori, MD $^{6}$

\begin{abstract}
This article is a continuation of previous reviews about the appropriate method for long-segment tracheal reconstruction. We attempted to cover the most recent, successful and promising results of the different solutions for reconstruction that are rather innovative and suitable for imminent clinical application. Latest efforts to minimize the limitations associated with each method have been covered as well. In summary, autologous and allogenic tissue reconstruction of the trachea have been successful methods for reconstruction experimentally and clinically. Autologous tissues were best utilized clinically to enhance revascularization, whether as a definitive airway or as an adjunct to allografts or tissue-engineered trachea (TET). Allogenic tissue transplantation is, currently, the most suitable for clinical application, especially after elimination of the need for immunosuppressive therapy with unlimited supply of tissues. Similar results have been reported in many studies that used TET. However, clinical application of this method was limited to use as a salvage treatment in a few studies with promising results. These results still need to be solidified by further clinical and longterm follow-up reports. Combining different methods of reconstruction was often required to establish a physiological rather than an anamical trachea and have shown superior outcomes.
\end{abstract}

Keywords: tracheal transplantation, tissue-engineered trachea, tracheal replacement, airway transplantation, tracheal substitutes, airway reconstruction

${ }^{1}$ Cardiothoracic Surgery Division, University of Alabama at Birmingham, Birmingham, Alabama, USA

${ }^{2}$ Thoracic Surgery, Faculty of Medicine, Ain Shams University, Cairo, Egypt

${ }^{3}$ Cardiothoracic Surgery, Faculty of Medicine, Assiut University, Assiut, Egypt

${ }^{4}$ Thoracic Surgery, Faculty of Medicine, Ain Shams University, Cairo, Egypt

${ }^{5}$ Cardiothoracic Surgery Division, University of Alabama at Birmingham, Birmingham, Alabama, USA

${ }^{6}$ Thoracic Surgery, Faculty of Medicine, Ain Shams University, Cairo, Egypt

Received: October 18, 2016; Accepted: November 18, 2016

Corresponding author: Ahmed A. Abouarab, MD, MRCS. 1343

14th Ave South, Birmingham, Alabama, 35205, USA

Email: abouarab@uab.edu, anter4@hotmail.com

(C)2017 The Editorial Committee of Annals of Thoracic and Cardiovascular Surgery. All rights reserved.

\section{Introduction}

Main problem and pathological background

The surgical treatment of extensive tracheal lesions where tracheal resection with primary anastomosis is not possible remains an unsurpassed problem. Extensive lesions can be defined as defects, regardless of the etiology, that are longer than half of the trachea in adults or one third in children. ${ }^{1)}$ Thus, a substitute is required. The ideal substitute for the trachea should be; laterally rigid, longitudinally flexible, and has a surface of ciliated respiratory epithelium. If artificial prosthetics are to be utilized, these foreign materials should be biocompatible, non-toxic, non-immunogenic, and non-carcinogenic. Clinically, the substitute should resist dislocation, erosion, 
stenosis and accumulation of secretions, and bacterial colonization. ${ }^{2)}$ The various tracheal substitutes and techniques of reconstruction were analyzed by Grillo, who classified them into five categories: foreign materials, non-viable tissues, autogenous tissues, tissue engineering, and tracheal transplantation. ${ }^{3)}$ Many clinical and biological efforts have been invested to assess these methods, paving the way for the current innovative and promising solutions that are anticipated to be applied on a larger scale soon. Previous successful studies have been covered in other reviews. ${ }^{1,4)}$ In this review, we aim to cover the most recent clinical updates about this subject with emphasis on the most recent innovative methods and endeavors to overcome their limitations.

\section{Tracheal Reconstruction with Autologous Tissues}

\section{Autogenous graft}

Different autogenous tissues were used for repair of tracheal defects either as patches or in tubular forms. More recent clinical studies used other tissues with even better outcomes, mainly; bronchial patches, dermal grafts, pericardium, and aortic grafts. ${ }^{1)}$ In 2000, Martinod et al. ${ }^{5,6}$ succeeded in using a thoracic aortic patch with temporary stenting experimentally. His studies and case reports were innovative, not only for taking advantage of two different methods of tracheal repair, but also due to his finding that metaplasia of aortic tissue into a well-differentiated tracheal tissue can be achieved. This study and its follow-up report in 2003 have revolutionized the research to find an appropriate substitute and opened the door for trials of in situ regeneration and the different modalities of tissueengineered trachea (TET) replacement.

This was further supported by Dodge-Khatami's report of success in using a carotid artery patch in children. ${ }^{7)}$ This concept entails that foreign materials do not function as a definitive substitute for the trachea, but a rather temporary method to prevent collapse of the lumen, whereas the autogenous graft grow to its full strength. In 2009, in an experimental study by Anoosh et al., ${ }^{8}$ based on Martinod's reports, they used the abdominal aorta to repair cervical trachea leaving the posterior membranous layer intact without stenting. This study confirmed the previous results and showed the advantages of this method, namely; less immunogenicity, less vascularity, and absence of mucous secretions or peristalsis. However, they suggested that its application is not clinically practical, and proposed the use of an aortic allograft as a more reasonable option for humans.

\section{Autogenous flaps}

Autologous tissue repair of tracheal defects is preferred over allogenic tissues. However, these tissues still require constant blood supply and mucosal lining for intact cough reflex. Flap tissues are characterized by an independent blood supply and better outcomes in terms of wound healing. Thus, flap tissues are more reliable than autogenous grafts. Typically, flaps are used to treat irradiated or heavily scarred areas due to poor blood supply. ${ }^{1)}$ The different types of flaps have been experimentally used: local, regional, and free flaps. Clinically, the following were the most successful flaps: cutaneous chondromucosal forearm tubular flaps, compound tracheal segments vascularized by sternocleidomastoid muscle, deltopectoral flap with costal cartilage and palatal mucosal grafts, and pectoralis major myocutaneous flap with costal cartilage. ${ }^{1)}$

In 2006, Yu et al. ${ }^{9)}$ had a remarkable trial, when they used a radial forearm free flap in combination with polymex mesh and hemi shield vascular graft to create a patent airway with normal swallowing and voice. However, all of these procedures required lengthy multi-stage procedures, and despite the promising results of using flaps in reconstruction of the trachea, it is not suitable for the treatment of cancer patients particularly, which is one of the major causes of large segment tracheal defects.

In a remarkable case report by Ren et al., ${ }^{10)}$ they repaired a long-segment tracheal defect in a 48-year-old man with extended bronchial flap of right upper and main bronchus and tracheoplasty. In this surgical technique, the tension was dispersed to a wide range of anastomosis. Thus, lowering the risk of dehiscence was effectively achieved. They also believe that muscle enwrapping (was not performed in this case) would avoid ischemia of the flap and subsequent fistula. Nevertheless, there has been some debate about the benefit of sacrificing the right upper lobe to counterbalance the radical resection of malignant tumors as in this case. In a report by Grillo, ${ }^{11)}$ he has established that radical resection of extensive tracheal tumors offers better survival chances than palliative treatment. Particularly, for giant tumors with obstructive symptoms.

\section{Tracheal Transplantation and Reconstruction of Tracheal Defects with Allogenic Tissues}

\section{Devascularized transplantation}

In the last century, multiple studies concluded that devascularized grafts are not a reliable method of treatment 
with only minimal success using short segments. ${ }^{12,13)}$ Rejection was another problem that needed to be addressed. To that end, immunosuppressive therapy has been tried to tackle this problem. However, these allografts resulted in extensive fibrosis. ${ }^{13)}$ These failures triggered attempts to preserve the tracheal tissue and remove the antigenic sources. First, the main antigenic source of the transplanted tissue was determined by Beigel et al. ${ }^{14)}$ and Bujia et al. ${ }^{15)}$ They identified that the mucosa is the major antigenic source. Subsequently, many experimental trials involved de-epithelialization, merthiolate-treating, and cryopreservation of the allografts. However, these trials have resulted in failures with fibrosis, stenosis, or/and necrosis. ${ }^{1)}$

\section{Vascularized tracheal allografts}

Tracheal grafts can be directly vascularized by creating anastomoses or with flaps. In early trials of vascularized tracheal allotransplantation, indirect omental revascularization of allotransplants promoted faster results in short tracheal segments. ${ }^{16)}$ However, the omentum alone was not sufficient to neither support longer tracheal allografts nor maintain chondrocytes. ${ }^{17)}$

Yokomise et al. ${ }^{18)}$ attempted to structurally modify the graft by introducing a graft split in the middle by an omental circumferential segment. This has prevented central graft necrosis and the graft revascularized without stenosis or necrosis. This was further modified by Murai and colleagues. ${ }^{19)}$ when they removed the cartilage rings in order to increase contact to improve blood supply and re-epithelialization. In an attempt to overcome rejection, cryopreservation of the allografts was used and resulted in success even without immunosuppression. ${ }^{1820)}$ The benefit from cryopreservation is that it inhibits immunogenicity while maintaining structural integrity. Thus, cryopreservation reduces acute rejection and permits early revascularization. Nevertheless, on the long term, chronic rejection has been reported causing vascular occlusion and atrophy. ${ }^{21)}$

\section{Aortic allograft}

In 2005, Martinod et al. ${ }^{22)}$ attempted tracheal replacement with a fresh aortic allograft (FAA). In this experiment, the descending thoracic aortic segments were transplanted in the place of $8-\mathrm{cm}$ tracheal defects supported by a silicone stent without immunosuppression. Evidence of progressive metaplasia was seen in all specimens. This study confirmed the value of using aortic allografts as a tracheal substitute. Interestingly, this study has also showed that the neo-cartilage developed near normal tracheal rings and a posterior membrane which emphasize the diversity of the tissue transformation processes that can be achieved under appropriate conditions.

In 2009, to facilitate clinical application of the aortic allografts, the same team assessed the best preservation method for aortic allografts. As FAA are not readily available, especially, in emergency situations, they thought that it is more convenient to have allografts preserved in tissue banks. However, at that time little literature about whether aortic metaplasia into tracheal tissue would still occur with preserved allografts. Thus, they conducted this study to assess the use of cryopreserved, decellularized, or glutaraldehyde-treated aortic allografts as tracheal substitutes. These experiments confirmed that decellularized-allografts and glutaraldehyde-treated grafts gave disappointing results. Thus, the authors did not recommend these methods for allograft preservation in clinical use. They thought that cryopreservation of allografts represent the most promising solution. On the other hand, as decellularized aortic allograft did not allow regeneration of a tracheal conduit, they believe that the donor aortic cells are important for the regeneration. They suggested this phenomenon by delivering signals upon death leading stem cells to the graft or recipient cells having an essential contribution to this process. ${ }^{23)}$ In this view, they suggested that the inflammatory response is fundamental for tracheal regeneration based on a previous study. ${ }^{24)}$

In 2010, continuing to their previous efforts in this field, this research team attempted to use arterial allografts for airway transplantation for bronchial replacement. Thoracic aortic grafts were used, as well as common brachiocephalic trunks and descending aortas. Clinically, there were no perioperative mortalities. However, atelectasis developed in two animals, and was successfully treated by aspiration. Thus, this study further confirmed previous findings. ${ }^{25}$

In 2012, Kim et al. ${ }^{26)}$ reported their use of aortic allografts based on the studies by Martinod's team. In their study, they compared the results for both FAA and cryopreserved aortic allografts (CAA). This study showed that FAA and CAA did not show any evidence of acute or chronic rejection without immunosuppression. Tracheal components including cartilage regenerated as well in the graft. However, newly-formed cartilage was immature and its function was negligible. Cartilage was dispersed rather than ring shaped. This resulted in 
diminished rigidity that is required as a functional trachea. Consequently, the authors of this study suggested that human subjects may be required to have a tracheal stent placed for an extended period after transplantation.

\section{Clinical application of allografts}

Delaere et al. ${ }^{27)}$ reported a case of 55-year-old status post tracheotomy with a long history of tracheal stenosis and stents placement associated with multiple complications since 2006. Given her poor quality of life, the authors decided to perform tracheal allotransplantation. Grounded on success of similar technique in previous experimental studies, they planned to place an 8-cm-long tracheal allograft in the recipient's forearm to permit revascularization for later use in the defect under immunosuppression. Interestingly, in their study the viability of the tracheal cartilage was maintained, even after elimination of immunosuppression. This supports the hypothesis that allografts with intact cartilage are resistant to rejection and that the donor tracheal cartilage remains viable when surrounded by vascularized recipient tissue.

Since then, the same team had four additional patients who had tracheal allotransplantation. They aimed to reduce the time between forearm implantation and orthotopic transplantation, and to determine a safe protocol for tapering of immunosuppressive therapy. Similarly, after forearm implantation, all transplants became fully revascularized followed by withdrawal of immunosuppression after graft implantation. In these cases, the mucosal lining by recipient cells to compensate for the necrosis of the donor mucosa was not sufficient. Thus, partial loss of the allotransplant occurred in some patients. The authors suggested that the trachea may be used as a composite tissue allotransplant with heterotopic revascularization in the forearm; however, maximizing cell repopulation in the recipient may be important in maintaining the viability of the transplant after stopping immunosuppression. ${ }^{28)}$

In 2014, Zanetta et al., ${ }^{29)}$ attempted to reconstruct the larynx and trachea with a 10-cm CAA as a last resort after complicated cricotracheal resection in a 4-year-old girl with tracheostomy and laryngeotracheal stenosis after prolonged intubation. The graft was further augmented with a thymopericardial-flap and a nasotracheal tube was left in place. After 20 days, a tracheotomy cannula and suprastomal endoprosthesis were placed. Without immunosuppression, a progressive transformation of the white-internal surface of the graft into a healthy vascularized conduit was achieved with no signs of rejection. However, there was no clear evidence of cartilage rings formation in the walls at the time of the report. Thus, it was not known if the patient will be decannulated. A follow-up report for this case would be valuable for determination of the applicability of this procedure especially in non-emergent situations.

In another clinical study, Farwell et al. ${ }^{30)}$ attempted laryngotracheal transplantation in a 51-year-old with prior kidney-pancreas transplant presenting with laryngotracheal stenosis and is already on immunosuppressive therapy. On follow up, the patient did not show any clinical or pathologic signs of rejection. The trachea and subglottic larynx remained patent. However, the glottic airway was limited and precluded decannulation. This is another study that demonstrated a successful laryngotracheal transplant. The authors of this study suggested that, with proper surgical technique, these complications could be overcome.

In 2014, Xu et al. ${ }^{31)}$ reviewed the results of two-stage tracheal allograft reconstruction for long tracheal defects with the use of greater omentum. Tracheal allograft transplantation was performed in three patients. In the first stage, a $6 \mathrm{~cm}$ segment of donor trachea was excised and wrapped in the recipient's greater omentum and implanted for revascularization in the abdomen. In the second stage, the tracheal allograft and omental pedicle were orthotopically transplanted to the cervical part of the trachea. Clinically, no major postoperative complications were observed. Postoperative bronchoscopy showed a wellhealed tracheal anastomosis, with no signs of bronchiolitis obliterans. At the beginning of the second stage, a transverse section of the donor trachea showed a stable cartilage framework, and histologic section showed complete cartilage rings covered by intact respiratory epithelium. This study has established the concept of clinical tracheal allograft transplantation with omental wrapping in the abdomen in a two-stage procedure.

\section{Tissue-engineered trachea}

In order to construct a TET, scientists need to provide reproducible cell lines that have the potential for generating respiratory epithelium and a scaffold for reproduction of the structural frame. ${ }^{32)}$

Recently, Park et al. ${ }^{33)}$ devised a novel TET with mechanical behavior similar to native trachea using a hollow-bellows scaffold as a framework and described a reliable method for 3D-printing of monolithic bellows scaffold. They also functionalized gelatin sponge to allow for sustained release of transforming growth factor 
beta (TGF- $\beta$ ) for cartilage regeneration and confirmed that functionalized gelatin sponge induces cartilaginous tissue formation in vitro. They created a TET by assembling functionalized gelatin sponges with chondrocytes seeding onto the grooves of bellows scaffold.

Go et al. ${ }^{34)}$ suggested that seeding of both epithelial cells and mesenchymal stem cell-derived chondrocytes is necessary for better graft survival.

Luo et al. ${ }^{35)}$ tested the long-term reconstruction using a process involving culture before implantation, followed by in vivo maturation, in addition to vascularization of TET grafts and stenting. Eventually, long-term survival of animals was achieved.

\section{Clinical application of TET}

In the past decade, attempts to test the TET in clinical settings have been documented with promising results. In 2004, Macchiarini et al. ${ }^{36)}$ reported an experience of using a tissue-engineered tracheal patch made of autologous muscle and fibroblasts seeded on a collagen scaffold of a decellularized porcine proximal jejunum segment. The bioartificial trachea was used to close the tracheal defect in a 58-year-old man. Endoscopic examination confirmed the absence of air leakage and graft coverage with recipient's ciliated respiratory epithelium. Twelve weeks postoperatively, the bio-artificial patch was functionally and morphologically merged with adjacent tissues without chronic inflammation, infection, or granulation tissue. This case report proved the value of tissue engineering in reconstruction of tracheal defects.

In 2005, Omori et al. ${ }^{37)}$ constructed a TET to repair the trachea of a 78-year-old woman using a scaffold of Marlex porous mesh tube covered by a collagen sponge made from porcine dermal atelocollagen. The collagen sponge was intended to enhance tissue invasion into the Marlex mesh scaffold and thus, assist in re-epithelialization of the lumen. Interestingly, epithelialization of the tracheal lumen was achieved at 2 months and was completed 2 years later without any complications.

In 2008, the first entirely tissue-engineered airway replacement in a human being was successfully performed using a bioengineered human trachea to restore lung function of a patient with end-stage left-main bronchus malacia. ${ }^{38)}$ Airway from a deceased donor was used. In a special bioreactor, the matrix was re-seeded with expanded and differentiated autologous epithelial cells and chondrocytes of mesenchymal-stem cell origin, and then implanted. At 4 months after surgery, the patient was well with normal lung function without immunosuppression.
However, several points needed to be addressed: the possibility of maintaining a viable, re-cellularized, and functional airway after implantation, long-term stability of the decellularized natural matrix, and the fate and tumorigenic risks associated with the stem cells use. ${ }^{37)}$

Thus, a follow-up report was needed. Consequently, the authors of this study reported the 5-year follow-up results. ${ }^{39)}$ The quality of life assessment was affected only by endoscopies. However, the patient always defined her health as "excellent" or "very good". During the 5 years she has never been prevented from her usual activities. While the graft was functioning well until 6 months after surgery, pulmonary function tests remained normal until 44 months postoperatively. She required multiple dilations and stenting due to stenosis and accumulation of secretions. However, the patient did not report any restriction in day-to-day activities or down-grade her perception of quality of life. During the 5 years of follow up, no stenosis developed at the distal bronchial anastomosis and the rest of the graft remained patent. Completely recellularized airway was developed with morphology very similar to the native airway without signs of immunogenicity or rejection. These results confirmed the safety of clinical application of this previously tested method. ${ }^{40)}$

In 2011, Jungebluth et al. ${ }^{41)}$ reported their experience of use of a tailored bioartificial nanocomposite seeded with marrow mononuclear cells via a bioreactor to replace tracheal defect in a 36-year-old male patient, previously treated with debulking surgery and radiation, presenting with recurrent primary cancer of the distal trachea and main bronchi. Aside from a brief episode of pneumonia which was successfully treated, the patient was doing well and discharged to the referral hospital 1 month after surgery. After 2 months, biopsy proved epithelialization and angiogenesis without infection. The patient was discharged and resumed his regular activities. At 5 months, the patient was asymptomatic with an almost normal airway. This study provided evidence that organ regeneration is feasible without the long process of decellularization and the need for a donor organ. This concept facilitates the clinical application of this method and makes it less time consuming.

In 2012, Elliot et al ${ }^{42)}$ reported a two-year follow up of the use of a stem-cell-based, tissue-engineered transplant in a 12-year-old boy born with long-segment congenital tracheal stenosis and pulmonary sling. They harvested a cadaveric trachea and used it as a decellularized scaffold. After exposure to granulocyte colony stimulating factor, 
bone marrow mesenchymal stem cells were collected and placed on to the scaffold mixed with patches of recipient's epithelium. Then, they used topical human recombinant erythropoietin to encourage angiogenesis, and TGF- $\beta$ to support chondrogenesis. They continued giving intravenous human recombinant erythropoietin, postoperatively. After 6 months, the graft was stable, the patient's airway was patent, and the patient had returned to the school. At fifteen months, complete epithelialization was achieved by ciliated respiratory epithelial cells. At 18 months, he underwent the last fluoroscopic balloon dilatation, and a previously malacic segment had developed rigidity that he did not need any further admissions to hospital. After 2 years, the patient was well, active and had grown $11 \mathrm{~cm}$ and $5 \mathrm{~kg}$ since graft implantation with no evidence of rejection of the graft by a screening for anti-HLA antibodies.

In 2014, the same team reported the 4-year follow up of the same patient with aim to address the following important aspects: the capacity of the transplanted airway for growth in children, the long-term graft remodeling and continuity of the regenerated epithelium immunity tolerance, the quality of life, the timeframe of events, and the cost necessary for application. To that end, they found that the child's height grew $15 \mathrm{~cm}$ to $168 \mathrm{~cm}$ and his weight increased $21 \mathrm{~kg}$ to $58 \mathrm{~kg}$. The patient required multiple hospital admissions for secretions and stenoses that required multiple dilations and stent placements. After the first 6 months, the airway was stable and the patient returned to school and the frequency of interventions decreased until late 2013 when tracheal stent infection and stenosis occurred. However, after dilatation, the child was recovered and returned to school again. The quality of life assessment was consistent with a child suffering from a complex chronic disease. The authors suggested that this might have been due to other comorbidities (spastic diplegia and low physical subscale). The total cost of the procedure and clinical care was US $\$ 565414$ with the initial hospital admission accounting for $76 \%$ of the total. This cost was higher than other forms of management. However, only a small number of patients with airway stenosis will require this procedure. They expect that this cost will even further decline with improvements in techniques and complications management, resulting in shorter hospital stay. Using a TET, in this case, resulted in a 4-year survival. Functionally, the child grew, continued regular activities and was free of interventions for long time. Compared to conventional treatment, the timing and frequency of interventions during the whole process was the same. $\left.{ }^{43}\right)$
Berg et al. ${ }^{44)}$ reported a case of tracheal reconstruction with a detergent-prepared 3-dimensional scaffold of a human cadaveric trachea. After repopulation with autologous stem cells, it was used in a 76-year-old patient with tracheo-laryngeal stenosis. The postoperative course was complicated with fungal infection and treated well. However, the patient passed away, after 23 days, due to cardiac arrest. By which time, the tracheal transplant was patent and stable with intact anastomoses. Histopathological results of the transplanted tracheal graft during autopsy showed a squamous but not ciliated epithelium and normal connective tissue components. The authors of this study suggested that further preclinical studies are required.

In 2015, Steinke et al. ${ }^{45)}$ reported a 2.5 -year follow up of integration of a tissue-engineered airway patch (from porcine jejunal segments with their mesentery) to replace the membranous part of the trachea in a 26-year-old male suffering from an extensive tracheoesophageal defect. Biopsy analysis showed integrity and tissue reorganization; the surface of the airway was covered with respiratory epithelium indistinguishable from native epithelium. Authors of this study suggested that the capillary network of the transplant contributed to tissue ingrowth and healing without scar tissue formation or collapse. However, as mentioned before, they emphasized on the disadvantage of this approach due to long generation time which was 6 weeks. Tables 1 and $\mathbf{2}$ summarize the different clinical studies, which utilized the tissue-engineering technique.

\section{Discussion}

Based on the previously mentioned studies, regenerating a tracheal substitute that is merely anatomically similar to the trachea was not sufficient to maintain the patients clinically for extended time without re-intervention. Thus, the function of a native trachea has to be reproduced as well.

Tracheal prosthesis and stents were associated with many complications when used for definitive reconstruction of the trachea. Despite that, they were indispensable to support the trachea while other definitive methods were being implemented. Thus, the best utilization for them, so far, is probably as a temporary adjunct with another more long-lasting treatment.

Autologous-tissue reconstruction of the trachea has been a successful method for reconstruction when applied experimentally. In addition, this method enhanced the revascularization process when used in combination with other methods of reconstruction. In spite of this, this 


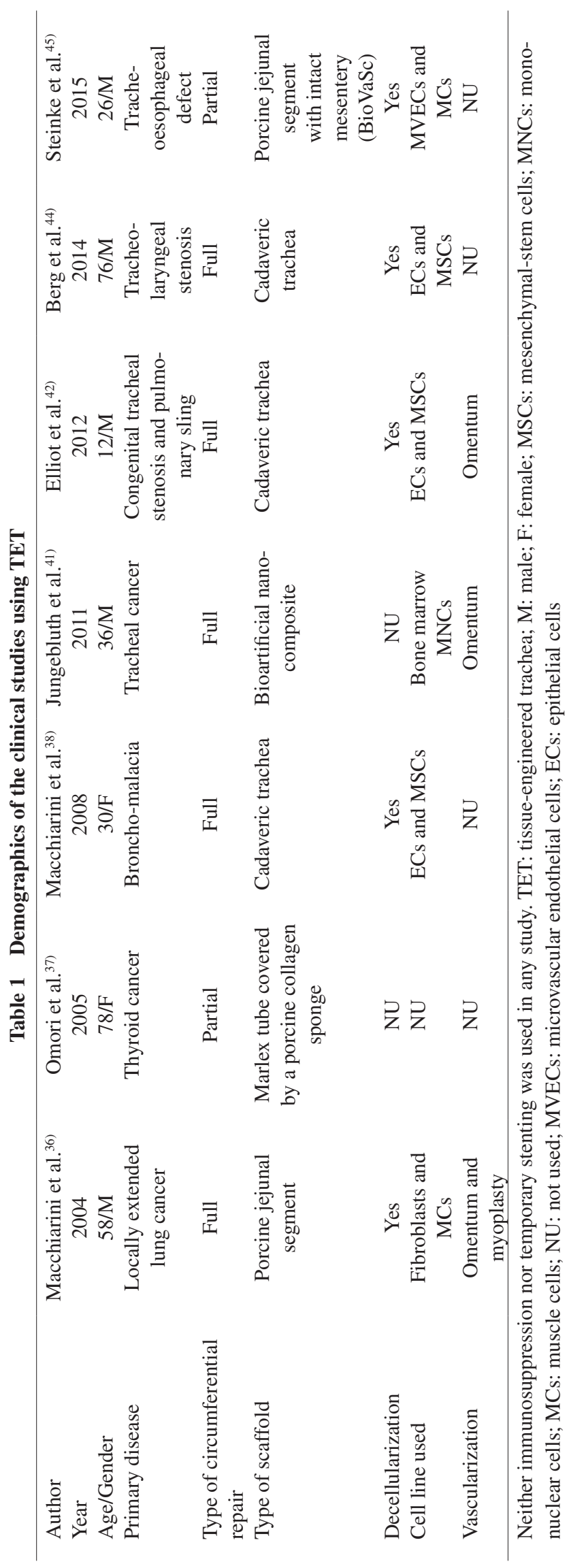

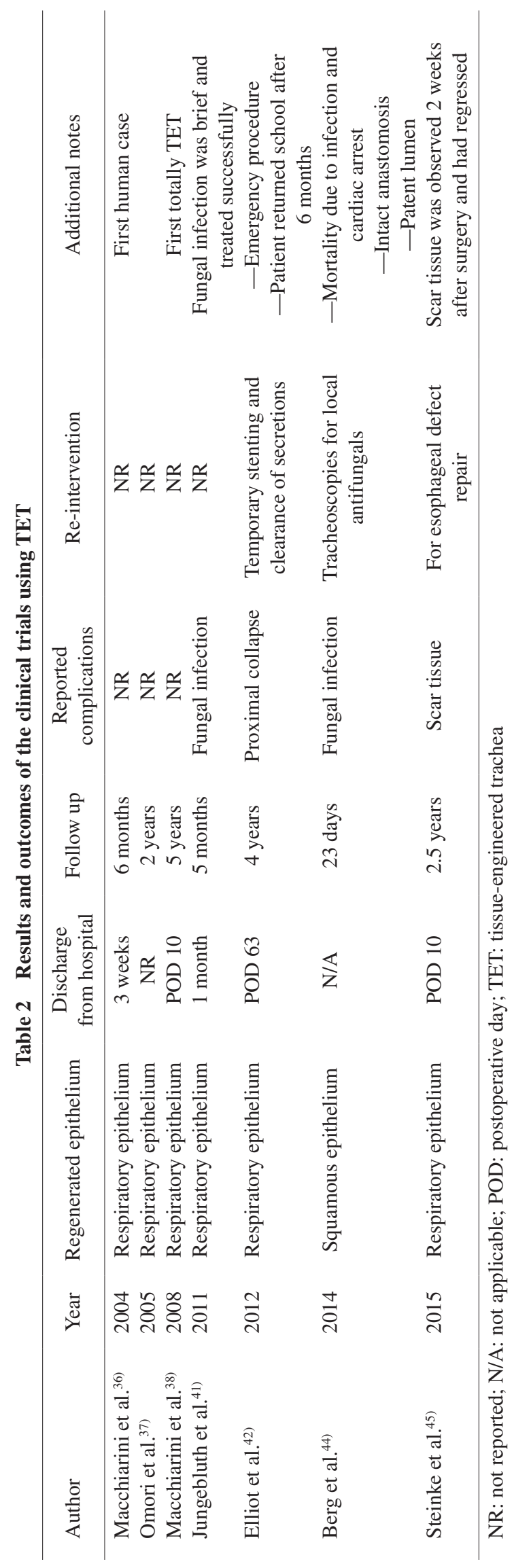

Ann Thorac Cardiovasc Surg Vol. 23, No. 2 (2017) 


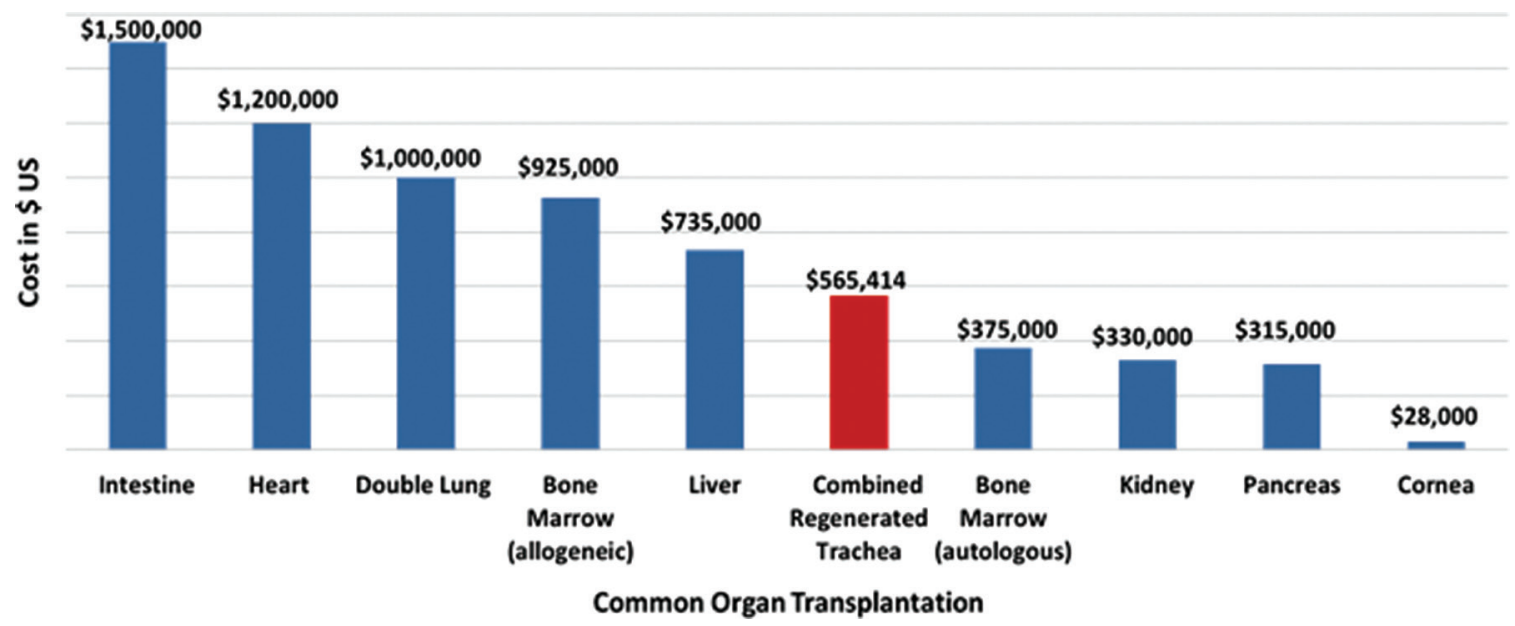

Fig. 1 Comparison between the cost of TET reported by Hamilton et al. ${ }^{43)}$ and the costs of common organ transplantation as reported in the Milliman, Inc. 2014 report. ${ }^{46)}$ TET: tissue-engineered trachea

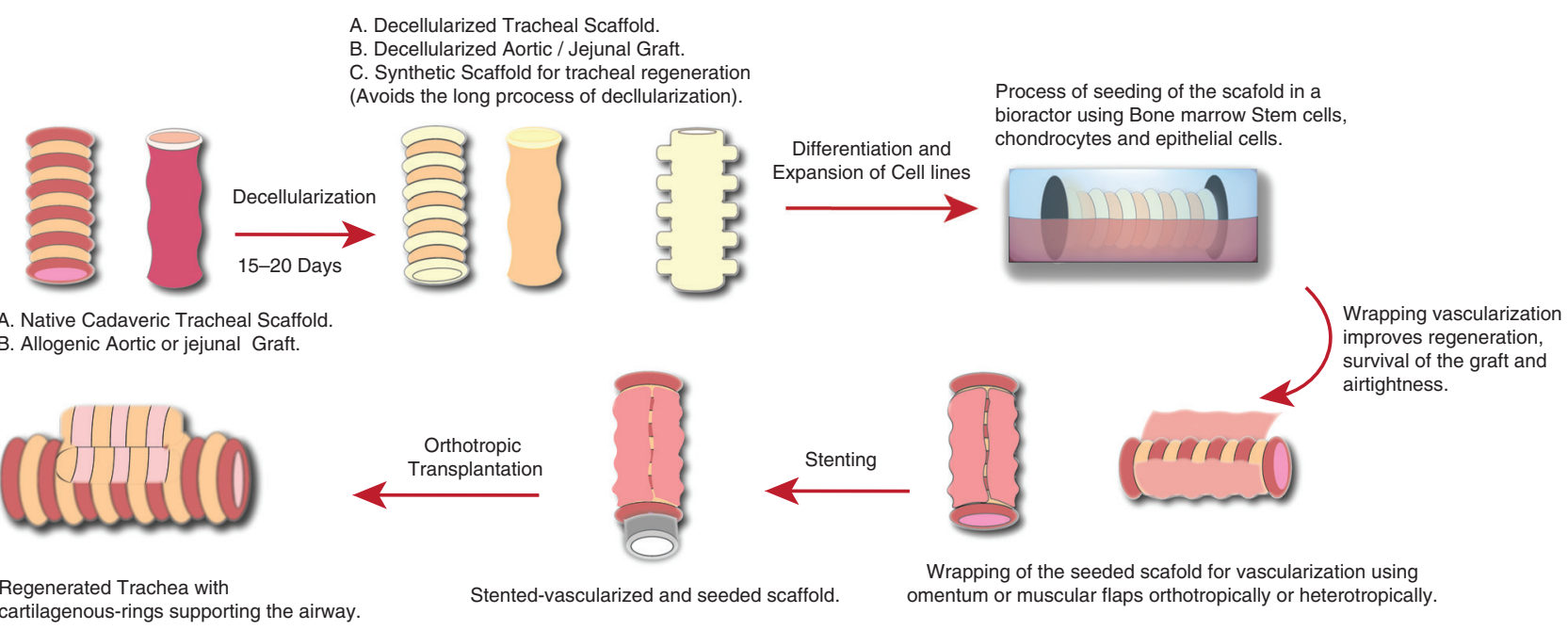

Fig. 2 The staged process of regenerating a tracheal substitute suitable for tracheal reconstruction.

method is not always practical for clinical application, especially in emergency settings. This is mainly related to the bioavailability of tissues, and perhaps the use of flaps is a more feasible option.

Allogenic-tissue reconstruction of the trachea showed excellent results both experimentally and clinically. The previous problem of immunogenicity and rejection has been greatly addressed. Interestingly, the need for immunosuppressive therapy has been eliminated, making this method one of the most promising methods for reconstruction of long segments of tracheal defects with unlimited supply of tissues. Additionally, combining this method with other methods of reconstruction has been associated with even better results.

Tissue-engineered tracheas used for reconstruction have shown promising results similar to that of allogenic-tissue repair. After many successful experimental methods, clinical application as last resort of treatment was associated with good results. This method holds the future for long-segment tracheal defects repair. However, these results still need to be solidified by further research, particularly, in clinical application and minimizing the costs and time of preparation. We compared the cost described by Elliot et al. ${ }^{42)}$ for this procedure with the costs of other organ transplantations in the USA reported in December 2014 by Milliman, Inc. ${ }^{46)}$ (Fig. 1). Despite that the current costs for this procedure is lower than other isolated organ transplantations such as intestinal and heart transplantation; we anticipate that this cost can potentially be decreased by development of technical experience and improved management of complications which accounted for a large portion of the total cost. 
We suggest that the concept of producing a scaffold for regeneration followed by seeding with bone marrow mesenchymal stem cells, epithelial cells and chondrocytes in a bioreactor is the most reliable method described for regeneration so far. Particularly, this method provides flexibility regarding the type of the scaffold used. Allogenic tracheal, aortic, or jejunal grafts can be used as well as different synthetic biocompatible materials, this variety is suitable for the different capacities of medical institutions. Perhaps synthetic scaffolds are more suitable for clinical application as it eliminates the time needed for decellularization of tissue grafts. However, to our knowledge, there are currently no studies that have compared the clinical outcomes of using the different scaffold options. Furthermore, wrapping the seeded scaffold with autogenous tissues such as omentum or muscular flaps, have improved vascularity, regeneration, and airtightness of the transplant. Following this with post-transplantation erythropoietin and TGF- $\beta$ were helpful in promoting angiogenesis and chondrogenesis as well. However, when the transplant was implanted in other parts of the body for revascularization prior to orthotropic transplantation this has resulted in extension of the time to definitive treatment and subjected patients to additional procedures. In this method, temporary stenting is helpful prior to orthotropic vascularization to support the transplant until it gains proper rigidity. Figure 2 summarizes the combined process for development of a tracheal substitute.

\section{Conclusion}

Combining the different methods of reconstruction showed superior outcomes to using any of them solely giving hope of cure to those patients with long tracheal defects. Until now, combining tissue-engineering techniques for seeding scaffolds followed by vascularization and temporary stenting showed promising clinical results. Perhaps investing more clinical research in this method and solutions to minimize the high cost will soon provide a reliable method for reconstruction.

\section{Disclosure Statement}

The authors declare that they have no conflicts of interest.

\section{References}

1) Kucera KA, Doss AE, Dunn SS, et al. Tracheal replacements: part 1. ASAIO J 2007; 53: 497-505.
2) Belsey R. Resection and reconstruction of the intrathoracic trachea. Br J Surg 1950; 38: 200-5.

3) Grillo HC. In: Surgery of the Trachea and Bronchi: Tracheal replacement. Hamilton, ON, Canada: BC Decker Inc; 2004: 839-54.

4) Haykal S, Salna M, Waddell TK, et al. Advances in tracheal reconstruction. Plast Reconstr Surg Glob Open 2014; 2: e178.

5) Martinod E, Zakine G, Fornes P, et al. [Metaplasia of aortic tissue into tracheal tissue. Surgical perspectives]. C R Acad Sci III 2000; 323: 455-60.

6) Martinod E, Seguin A, Pfeuty K, et al. Long-term evaluation of the replacement of the trachea with an autologous aortic graft. Ann Thorac Surg 2003; 75: 1572-8; discussion 1578.

7) Dodge-Khatami A, Nijdam NC, Broekhuis E, et al. Carotid artery patch plasty as a last resort repair for long-segment congenital tracheal stenosis. J Thorac Cardiovasc Surg 2002; 123: 826-8.

8) Anoosh F, Hodjati H, Dehghani S, et al. Tracheal replacement by autogenous aorta. J Cardiothorac Surg 2009; 4: 23.

9) Yu P, Clayman GL, Walsh GL. Human tracheal reconstruction with a composite radial forearm free flap and prosthesis. Ann Thorac Surg 2006; 81: 714-6.

10) Ren YJ, Zheng H, Shen LX, et al. A Novel technique of long-segment tracheal repair with extended bronchial flap of right upper and main bronchus plus tracheoplasty. Ann Thorac Surg 2015; 99: 2188-90.

11) Grillo HC, Mathisen DJ. Primary tracheal tumors: treatment and results. Ann Thorac Surg 1990; 49: 69-77.

12) Neville WE, Bolanowski PJ, Soltanzadeh H. Homograft replacement of the trachea using immunosuppression. J Thorac Cardiovasc Surg 1976; 72: 596-601.

13) Spinazzola AJ, Graziano JL, Neville WE. Experimental reconstruction of the tracheal carina. J Thorac Cardiovasc Surg 1969; 58: 1-13.

14) Beigel A, Steffens-Knutzen R, Müller B, et al. Tracheal transplantation. III. Demonstration of transplantation antigens on the tracheal mucosa of inbred rat strains. Arch Otorhinolaryngol 1984; 241: 1-8.

15) Bujia J, Wilmes E, Hammer C, et al. Tracheal transplantation: demonstration of HLA class II subregion gene products on human trachea. Acta Otolaryngol 1990; 110: 149-54.

16) Klepetko W, Marta GM, Wisser W, et al. Heterotopic tracheal transplantation with omentum wrapping in the abdominal position preserves functional and structural integrity of a human tracheal allograft. J Thorac Cardiovasc Surg 2004; 127: 862-7.

17) Li J, Xu P, Chen H, et al. Improvement of tracheal autograft survival with transplantation into the greater omentum. Ann Thorac Surg 1995; 60: 1592-6.

18) Yokomise $H$, Inui $K$, Wada $H$, et al. Long-term cryopreservation can prevent rejection of canine tracheal allografts with preservation of graft viability. J Thorac Cardiovasc Surg 1996; 111: 930-4. 
19) Murai K, Oizumi H, Masaoka T, et al. Removal of cartilage rings of the graft and omentopexy for extended tracheal autotransplantation. Ann Thorac Surg 1999; 67: 776-80.

20) Moriyama H, Sasajima T, Hirata S, et al. Revascularization of canine cryopreserved tracheal allografts. Ann Thorac Surg 2000; 69: 1701-6.

21) Mukaida T, Shimizu N, Aoe M, et al. Experimental study of tracheal allotransplantation with cryopreserved grafts. J Thorac Cardiovasc Surg 1998; 116: 262-6.

22) Martinod E, Seguin A, Holder-Espinasse M, et al. Tracheal regeneration following tracheal replacement with an allogenic aorta. Ann Thorac Surg 2005; 79: 942-8; discussion 949.

23) Seguin A, Radu D, Holder-Espinasse M, et al. Tracheal replacement with cryopreserved, decellularized, or glutaraldehyde-treated aortic allografts. Ann Thorac Surg 2009; 87: 861-7.

24) Takahashi K, Fukushima S, Yamahara K, et al. Modulated inflammation by injection of high-mobility group box 1 recovers post-infarction chronically failing heart. Circulation 2008; 118: S106-14.

25) Radu DM, Seguin A, Bruneval P, et al. Bronchial replacement with arterial allografts. Ann Thorac Surg 2010; 90: 252-8.

26) Kim DH, Choi CB, Yang WJ, et al. Tracheal replacement with fresh and cryopreserved aortic allograft in adult dog. J Surg Res 2012; 175: 199-206.

27) Delaere P, Vranckx J, Verleden G, et al. Tracheal allotransplantation after withdrawal of immunosuppressive therapy. N Engl J Med 2010; 362: 138-45.

28) Delaere PR, Vranckx JJ, Meulemans J, et al. Learning curve in tracheal allotransplantation. Am J Transplant 2012; 12: 2538-45.

29) Zanetta A, Cuestas G, Rodríguez H, et al. [Laryngotracheal reconstruction with cryopreserved aortic allograft as a salvage technique when cricotracheal resection complications occur in paediatrics]. Acta Otorrinolaringol Esp 2014; 65: 191-3. (in Spanish)

30) Farwell DG, Birchall MA, Macchiarini P, et al. Laryngotracheal transplantation: technical modifications and functional outcomes. Laryngoscope 2013; 123: 2502-8.

31) Xu L, Zhang S, Li J, et al. Human tracheal allotransplant with greater omentum for revascularization. Exp Clin Transplant 2014; 12: 448-53.

32) Doss AE, Dunn SS, Kucera KA, et al. Tracheal replacements: Part 2. ASAIO J 2007; 53: 631-9.
33) Park JH, Hong JM, Ju YM, et al. A novel tissueengineered trachea with a mechanical behavior similar to native trachea. Biomaterials 2015; 62: 106-15.

34) Go T, Jungebluth P, Baiguero S, et al. Both epithelial cells and mesenchymal stem cell-derived chondrocytes contribute to the survival of tissue-engineered airway transplants in pigs. J Thorac Cardiovasc Surg 2010; 139: 437-43.

35) Luo X, Liu Y, Zhang Z, et al. Long-term functional reconstruction of segmental tracheal defect by pedicled tissue-engineered trachea in rabbits. Biomaterials 2013; 34: 3336-44.

36) Macchiarini P, Walles T, Biancosino C, et al. First human transplantation of a bioengineered airway tissue. J Thorac Cardiovasc Surg 2004; 128: 638-41.

37) Omori K, Nakamura T, Kanemaru S, et al. Regenerative medicine of the trachea: the first human case. Ann Otol Rhinol Laryngol 2005; 114: 429-33.

38) Macchiarini P, Jungebluth P, Go T, et al. Clinical transplantation of a tissue-engineered airway. Lancet 2008; 372: 2023-30.

39) Gonfiotti A, Jaus MO, Barale D, et al. The first tissueengineered airway transplantation: 5-year follow-up results. Lancet 2014; 383: 238-44.

40) Baiguera S, Jungebluth P, Burns A, et al. Tissue engineered human tracheas for in vivo implantation. Biomaterials 2010; 31: 8931-8.

41) Jungebluth P, Alici E, Baiguera S, et al. Tracheobronchial transplantation with a stem-cell-seeded bioartificial nanocomposite: a proof-of-concept study. Lancet 2011; 378: 1997-2004.

42) Elliott MJ, De Coppi P, Speggiorin S, et al. Stem-cellbased, tissue engineered tracheal replacement in a child: a 2-year follow-up study. Lancet 2012; 380: 994-1000.

43) Hamilton NJ, Kanani M, Roebuck DJ, et al. Tissueengineered tracheal replacement in a child: a 4-year follow-up study. Am J Transplant 2015; 15: 2750-7.

44) Berg M, Ejnell H, Kovács A, et al. Replacement of a tracheal stenosis with a tissue-engineered human trachea using autologous stem cells: a case report. Tissue Eng Part A 2014; 20: 389-97.

45) Steinke M, Dally I, Friedel G, et al. Host-integration of a tissue-engineered airway patch: two-year follow-up in a single patient. Tissue Eng Part A 2015; 21: 573-9.

46) Hanson, SG. Bentley, TS. 2014 U.S. organ and tissue transplant cost estimates and discussion. Brookfield, WI. Milliman, Inc. http://www.milliman.com/ uploadedFiles/insight/Research/health-rr/1938HDP_ 20141230.pdf. Access Sept. 2016. 